\title{
Reconstruction of the Lower Extremity Using Free Flaps
}

\author{
Min Jo Kang, Chul Hoon Chung, Yong Joon Chang, Kyul Hee Kim \\ Department of Plastic and Reconstructive Surgery, Hallym University College of Medicine, Seoul, Korea
}

Background The aim of lower-extremity reconstruction has focused on wound coverage and functional recovery. However, there are limitations in the use of a local flap in cases of extensive defects of the lower-extremities. Therefore, free flap is a useful option in lower-extremity reconstruction.

Methods We performed a retrospective review of 49 patients (52 cases) who underwent lower-extremity reconstruction at our institution during a 10-year period. In these patients, we evaluated causes and sites of defects, types of flaps, recipient vessels, types of anastomosis, survival rate, and complications.

Results There were 42 men and 10 women with a mean age of 32.7 years (range, 3-72 years). The sites of defects included the dorsum of the foot (19), pretibial area (17), ankle (7), heel (5) and other sites (4). The types of free flap included latissimus dorsi muscle flap (10), scapular fascial flap (6), anterolateral thigh flap (6), and other flaps (30). There were four cases of vascular complications, out of which two flaps survived after intervention. The overall survival of the flaps was $96.2 \%$ (50/52). There were 19 cases of other complications at recipient sites such as partial graft loss (8), partial flap necrosis (6) and infection (5). However, these complications were not notable and were resolved with skin grafts.

Conclusions The free flap is an effective method of lower-extremity reconstruction. Good outcomes can be achieved with complete debridement and the selection of appropriate recipient vessels and flaps according to the recipient site.

Keywords Free tissue flap / Lower extremity / Microsurgery

Correspondence: Chul Hoon Chung

Department of Plastic and

Reconstructive Surgery, Hallym

University College of Medicine,

150 Seongan-ro, Gangdong-gu,

Seoul 134-701, Korea

Tel: +82-2-2224-2246

Fax: +82-2-489-0010

E-mail:c21ps@hanmail.net

No potential conflict of interest relevant to this article was reported.

Received: 29 May 2013 • Revised: 9 Jul 2013 • Accepted: 10 Jul 2013

pISSN: 2234-6163・ elSSN: 2234-6171 • http://dx.doi.org/10.5999/aps.2013.40.5.575 • Arch Plast Surg 2013;40:575-583

\section{INTRODUCTION}

In cases of defects of the lower extremities, there is a paucity of local soft tissue and blood supply. This poses challenging problems for surgeons. Among the methods for reconstructing defects of the lower extremities, there are direct closure, skin grafting, and local flaps including the muscle flap, cross-leg flap, and free flap [1]. The local flap has the disadvantage of limiting mobility. The cross-leg flap has disadvantages, too, not only in that it causes discomfort to patients because it restricts the movement of the legs, but also in that it requires a secondary operation to isolate the pedicle. The free flap has the disadvantage that it requires microsurgical techniques and good vascularity of blood vessels at the recipient sites, and it may prolong operation time. It is advantageous in that the flap itself has good vascularity, identical tissue can be easily obtained, there is almost no limitation on its use as far as size of the defect sites, and satisfactory cosmetic outcomes can be obtained. Pollak et al. [2] reported that a bet- 
ter prognosis would be achieved in reconstruction surgery using a free flap even in cases in which the reconstruction could be performed sufficiently using a local flap.

In this study, we analyzed cases in which free flaps were used for the reconstruction of lower extremity defects and discussed the treatment strategy with a review of the literature.

\section{METHODS}

In the current study, we performed a retrospective analysis of the medical records of 49 patients (52 cases) who underwent lower extremity reconstruction at our medical institution during a period ranging from 1996 to 2005 . The mean follow up period was 12.2 years (range, 7-17 years). Of these, three patients had defects concurrently at two different sites. Specifically, we reconstructed defects of the dorsum of both feet using latissimus dorsi muscle free flaps for both. In addition, we reconstructed the defects of pretibial soft tissue and the heel using a latissimus dorsi muscle free flap and a medial plantar sensory free flap, respectively. Furthermore, we reconstructed the defects of the tibia and heel using a fibular osteocutaneous free flap and a latissimus dorsi muscle free flap, respectively. In all, we performed a total of 52 cases of free-flap reconstruction of the lower extremity.

In most of the patients, an angiography of the lower extremities was performed approximately one week before the operation. This was performed for all the patients with diabetes mellitus or hypertension and, in particular, the elderly aged 60 years or older.

In the current study, we analyzed the factors that might affect the survival of the free flap, which include age; sex; smoking; systemic diseases such as diabetes mellitus, hypertension and vascular disease; the causes and sites of the defects; recipient vessels; and the methods of vascular anastomosis. Moreover, we also analyzed the survival and complications of the free flap. A statistical analysis was performed using SPSS ver. 20.0 (IBM Corp., Armonk, NY, USA). Data were analyzed using the Student's t-test for nonparametric variables, and chi-squared analysis and Fisher's exact test were used for categorical variables. A value of $\mathrm{P}<0.05$ was taken to indicate statistical significance.

\section{RESULTS}

\section{Baseline and demographic characteristics}

Our clinical series of patients was composed of 42 men and 10 women, showing a male predilection, whose mean age was 32.7 years old (range, 3-72 years old). There was 1 patient who solely had diabetes mellitus and three patients who solely had hypertension. In addition, there was 1 patient who concurrently had diabetes mellitus, hypertension, and atherosclerosis. Moreover, there was 1 patient who concurrently had diabetes mellitus and hypertension and 2 patients who concurrently had diabetes mellitus and atherosclerosis. Finally, there were 15 smokers.

The analysis of correlation between each risk factor and complication showed that the $\mathrm{P}$-values of sex $(\mathrm{P}=1.000)$, age $(\mathrm{P}=$ 0.209), diabetes mellitus ( $\mathrm{P}=1.000)$, hypertension $(\mathrm{P}=0.368)$, atherosclerosis $(\mathrm{P}=0.577)$, and smoking $(\mathrm{P}=0.400)$ exceeded 0.05 , and therefore were not statistically significant. For this reason, no correlation was found in this study between risk factors and complications.

\section{Causes of defects}

In our series, causative factors included traffic accidents in 20 patients, electrical burns in 11 patients, unstable scarring and scar contracture in 11 patients, industrial injury in 6 patients, Marjolin's ulcer in 2 patients, chemical burns in 1 patient, contact burns in 1 patient, and hardware exposure in 1 patient (Table 1 ).

\section{Sites of defects}

The sites of the defects included the dorsum of the foot in 19 patients, which was the most prevalent site, the pretibial area in 17 patients, the ankle in 7 patients, the heel in 5 patients, the plantar forefoot in 2 patients, the thigh in 1 patient, and the popliteal region in 1 patient (Table 2 ). Of these, 2 patients with defects on the dorsum of the foot and 10 with defects on the pretibial area had the concurrent presence of osteomyelitis. Therefore, these patients also underwent skeletal reconstruction.

\section{Types of free flap}

We selected flaps for lower extremity reconstruction taking into consideration the size of the soft tissue defects, the concurrent presence of bone defects and osteomyelitis, and the location and characteristics of recipient sites.

There were 10 patients who underwent lower extremity reconstruction using a latissimus dorsi muscle flap, the most widely used flap, 6 with a scapular fascial flap, 6 with an anterolateral

Table 1. Causes of defects of the lower extremities

\begin{tabular}{|lc|}
\hline Causes & No. of cases \\
\hline Traffic accident & 20 \\
Unstable scarring and scar contracture & 11 \\
Electrical burn & 10 \\
Industrial injury & 6 \\
Marjolin's ulcer & 2 \\
Chemical burn & 1 \\
Contact thermal burn & 1 \\
Hardware exposure & 1 \\
Total & 52 \\
\hline
\end{tabular}


thigh flap, 5 with a medial plantar sensory flap, 5 with a fibular osteocutaneous flap, 4 with a scapular flap, 3 with both a latissimus dorsi muscle and a scapular osteocutaneous flap, 2 patients with a scapular osteocutaneous flap, 2 with an iliac osteocutaneous flap, 2 with both a latissimus dorsi muscle and a serratus anterior muscle flap for which the thoracodorsal artery served as a pedicle, 2 with a latissimus dorsi musculocutaneous flap, 2 with a serratus anterior muscle flap, 2 with a parietotemporal fascial flap, and 1 with a rectus abdominis muscle flap (Table 3 ). The types of free flaps for each defect site are summarized in Table 4.

\section{Size of free flap}

The mean size of the free flap was $10 \mathrm{~cm} \times 16 \mathrm{~cm}$. The smallest free flap was $5 \mathrm{~cm} \times 6 \mathrm{~cm}$, which was used in a 5-year-old boy who underwent reconstruction of a left heel defect using a medial plantar sensory free flap. The largest one was the flap for which the thoracodorsal artery served as a pedicle and both the latissimus dorsi muscle $(15 \mathrm{~cm} \times 25 \mathrm{~cm})$ and serratus anterior muscle $(10 \mathrm{~cm} \times 15 \mathrm{~cm})$ were elevated. This flap was used for a 35-yearold man who underwent reconstruction of the dorsum of the left foot and the plantar region.

\section{Table 2. Sites of defects}

\begin{tabular}{|lc|}
\hline Sites of defects & No. of cases \\
\hline Foot dorsum & 19 \\
Pretibial area & 17 \\
Ankle & 7 \\
Heel & 5 \\
Plantar forefoot & 2 \\
Thigh & 1 \\
Popliteal area & 1 \\
Total & 52 \\
\hline
\end{tabular}

\section{Vascular anastomosis}

Recipient arteries include the anterior tibial artery in 20 patients, the dorsalis pedis artery in 14 patients, the posterior tibial artery in 11 patients, the popliteal artery in 3 patients, the medial plantar artery in 1 patient, the lateral plantar artery in 1 patient, the superficial femoral artery in 1 patient, and the peroneal artery in 1 patient (Table 5). With respect to the methods of arterial anastomosis, there were 36 who received an end-to-end anastomosis and 15 patients who received an end-to-side anastomosis. The remaining 1 patient had a diabetic foot, for whom the dorsum of the right foot was reconstructed using a parietotemporal fascial flap. This patient had underlying diseases such as diabetes mellitus, hypertension, and atherosclerosis. Therefore, the patient had poor vascularity around the recipient site and had to receive a vein graft (Table 6). All the end-to-end anastomoses were performed on undamaged arteries at the recipient sites.

\section{Table 3. Types of free flaps}

\begin{tabular}{|lc|}
\hline Type of free flap & No. of cases \\
\hline Latissimus dorsi muscle flap & 10 \\
Scapular fascial flap & 6 \\
Anterolateral thigh flap & 6 \\
Medial plantar sensory flap & 5 \\
Fibular osteocutaneous flap & 5 \\
Scapular flap & 4 \\
Latissimus dorsi muscle and scapular & 3 \\
$\quad$ osteocutaneous flap & 2 \\
Scapular osteocutaneous flap & 2 \\
lliac osteocutaneous flap & 2 \\
Latissimus dorsi and serratus anterior muscle flap & 2 \\
Latissimus dorsi musculocutaneous flap & 2 \\
Serratus anterior muscle flap & 2 \\
Parietotemporal fascial flap & 1 \\
Rectus abdominis muscle flap & 52 \\
Total & \\
\hline
\end{tabular}

Table 4. Types of free flaps depending on the defect site

\begin{tabular}{|c|c|c|c|c|c|c|c|c|c|c|c|c|c|c|c|}
\hline \multirow{3}{*}{ Defect site } & \multicolumn{15}{|c|}{ Types of free flaps } \\
\hline & \multicolumn{4}{|c|}{$\begin{array}{c}M \\
(n=15)\end{array}$} & \multirow{2}{*}{$\begin{array}{c}\begin{array}{c}M C \\
(n=2)\end{array} \\
L D\end{array}$} & \multicolumn{2}{|c|}{$\begin{array}{c}F \\
(n=8)\end{array}$} & \multicolumn{2}{|c|}{$\begin{array}{c}F C \\
(n=10)\end{array}$} & \multicolumn{4}{|c|}{$\begin{array}{c}\text { OC } \\
(n=12)\end{array}$} & \multirow{2}{*}{$\begin{array}{c}M P \\
-(n=5)\end{array}$} & \multirow{2}{*}{ Total } \\
\hline & LD & $\mathrm{LD}+\mathrm{SA}$ & SA & RA & & SC & PT & ALT & SC & $\mathrm{FI}$ & $L D+S C$ & IA & SC & & \\
\hline Foot dorsum & 4 & 1 & 1 & - & - & 5 & 2 & 2 & 2 & - & - & - & 2 & - & 19 \\
\hline Pretibial area & 1 & 1 & - & 1 & 1 & - & - & 2 & 1 & 5 & 3 & 2 & - & - & 17 \\
\hline Ankle & 2 & 1 & 1 & - & - & 1 & - & 2 & 1 & - & - & - & - & - & 7 \\
\hline Heel & 2 & - & - & - & - & - & - & - & - & - & - & - & - & 3 & 5 \\
\hline Plantar forefoot & - & - & - & - & - & - & - & - & - & - & - & - & - & 2 & 2 \\
\hline Thigh & - & - & - & - & 1 & - & - & - & - & - & - & - & - & - & 1 \\
\hline Popliteal area & 1 & - & - & - & - & - & - & - & - & - & - & - & - & - & 1 \\
\hline Total & 10 & 2 & 2 & 1 & 2 & 6 & 2 & 6 & 4 & 5 & 3 & 2 & 2 & 5 & 52 \\
\hline
\end{tabular}




\section{Table 5. Types of recipient vessels}

\begin{tabular}{|lc|}
\hline Type of recipient vessel & No. of cases \\
\hline Anterior tibial artery & 20 \\
Dorsalis pedis artery & 14 \\
Posterior tibial artery & 11 \\
Popliteal artery & 3 \\
Medial plantar artery & 1 \\
Lateral plantar artery & 1 \\
Superficial femoral artery & 1 \\
Peroneal artery & 1 \\
Total & 52 \\
\hline
\end{tabular}

If we had to perform arterial anastomosis on the intermediate portion of major arteries in the lower extremities, we performed an end-to-side anastomosis. Two venous anastomoses were performed in each patient. That is, there were 31 patients for whom 2 donor site veins were anastomosed to the great saphenous vein and venae comitantes at the recipient sites. There were 13 patients for whom 2 recipient veins were anastomosed to the 2 venae comitantes at the recipient sites. There were 5 patients for whom 2 donor site veins were anastomosed to the lesser saphenous vein and the venae comitantes. There was 1 patient for whom 2 donor site veins were anastomosed to the popliteal vein and the venae comitantes at the recipient sites. There was one patient for whom 2 donor site veins were anastomosed to the great saphenous vein and the superficial femoral vein at the recipient sites. There was 1 patient for whom 2 donor site veins were anastomosed to the great saphenous vein and the lesser saphenous vein at the recipient sites. In patients who received a venous anastomosis of the popliteal vein or the superficial femoral vein, an end-to-side anastomosis was performed. In all the remaining recipient veins, an end-to-end anastomosis was performed. In 3 patients, vein grafting was performed.

\section{Complications and the survival of the free flap}

Of the 52 patients in total, 4 (7.7\%) presented vascular complications. These included 1 case of arterial thrombosis, 1 case of arterial spasm, and 2 cases of venous thrombosis. In 1 patient with arterial thrombosis, a scapular free flap was performed on the ankle. At the sites of anastomosis, the thrombus was removed and re-anastomosis was attempted, but the skin and adipose tissue had necrotized. Only the fascia survived. Therefore, the patient was treated with a full-thickness skin graft. The rectus abdominis muscle free flap was performed on the pretibial area in a 67-year-old man with arterial spasms and concurrent atherosclerosis. In this patient, there were findings that suggested arterial insufficiency. Grossly, there was no thrombus at the sites of arterial anastomosis. After the re-anastomosis, reperfusion was achieved, which led to the survival of the flap. In one
Table 6. Types of arterial anastomosis

\begin{tabular}{|lc|}
\hline Type of arterial anastomosis & No. of case \\
\hline End-to-end & 36 \\
End-to-side & 15 \\
Vein graft & 1 \\
Total & 52 \\
\hline
\end{tabular}

patient with venous thrombus, a latissimus dorsi muscle free flap was performed on the heel. In this case, there was thrombus at the sites of anastomosis. Therefore, the thrombus was removed and re-anastomosis was performed with vein grafting. Reperfusion was achieved and the flap survived. In another patient with venous thrombosis, a fibular osteocutaneous free flap was performed for the pretibial area. Thrombus was found at the sites of anastomosis. Following the removal of the thrombus, reanastomosis was performed with vein grafting, but only the skin flap necrotized. On postoperative day 20, the patient was treated with a new latissimus dorsi musculocutaneous free flap. In total, the overall survival rate of the free flaps was $96.2 \%(50 / 52)$.

There were a total of 19 cases (36.5\%) of other complications at recipient sites. These included 8 cases (15.4\%) of partial graft loss at sites where the muscle or fascial flap were inset, 6 cases (11.5\%) of partial necrosis of the flap, and 5 cases $(9.6 \%)$ of infection. Moreover, there were 19 cases (36.5\%) of other complications at the recipient sites, all of which were treated with a secondary skin graft.

The remaining 29 cases were treated without vascular complications or those at recipient sites. Out of the 52 patients, there were no complications at donor sites which required surgical treatment.

\section{Case 1}

A 28-year-old man had a $10 \mathrm{~cm} \times 15 \mathrm{~cm}$ soft tissue defect on the dorsum of the left foot caused by an electrical burn, and concurrently had bone exposure in the first and second toes. Following debridement, we performed arthrodesis of the first toe using a K-wire to prepare the recipient sites. The left scapular fascial free flap was elevated and then inset to the recipient sites. In this patient, an end-to-end anastomosis was performed between the circumflex scapular artery and the dorsalis pedis artery. In addition, two venae comitantes were anastomosed end-to-end to the great saphenous vein and the vena comitante of the dorsalis pedis artery. A split-thickness skin graft was performed over the flap. In the left scapular region, which served as the donor site, primary closure was performed (Fig. 1).

\section{Case 2}

A 17-year-old man presented necrosis of the right first and sec- 
Fig. 1. Defects of the dorsum of foot

A 28-year-old man with a defect of the dorsum of the left foot due to electrical burns. (A) Large soft tissue defect with necrosis of the extensor tendons and bone exposure of the 1st and 2nd toe following debridement. (B) Intraoperative photograph of the scapular fascial free flap insetting. (C) Photograph at postoperative month 7.
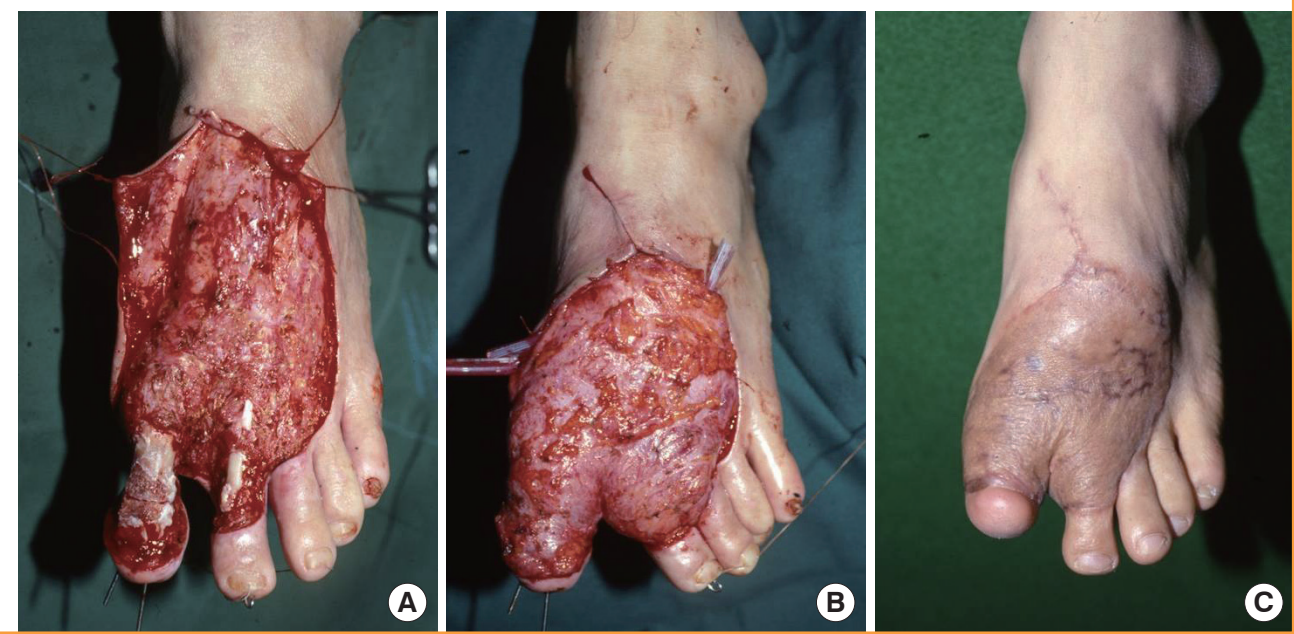

Fig. 2. Defects of the plantar forefoot

A 17-year-old man with a defect of the right plantar forefoot due to a traffic accident. (A) Large soft tissue defect with amputation of the 1st and 2nd toe following debridement. (B) Elevation of medial planter sensory free flap on the contralateral side. (C) Follow-up at postoperative month 3 .

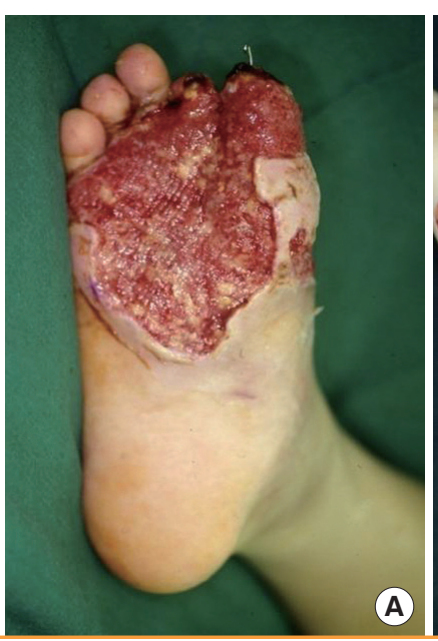

A
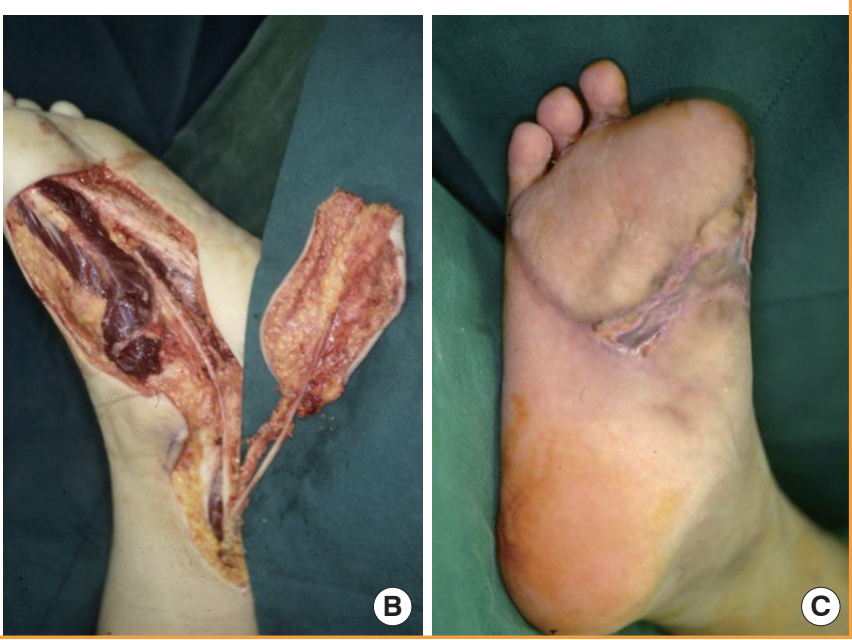

ond toes after sustaining a crushing injury in a traffic accident. In addition, the patient also had a $7 \mathrm{~cm} \times 11.5 \mathrm{~cm}$ soft tissue defect on the plantar forefoot. The necrotized first and second toes were amputated from the metatarsophalangeal joint. The recipient sites were prepared by debridement. The plantar forefoot comes into contact with the floor during walking. We therefore elevated the medial plantar sensory free flap on the contralateral side of the plantar region for the reconstruction procedure. The medial plantar artery distributed in the flap was anastomosed to the dorsal pedis artery of the recipient site. In addition, two venae comitantes were anastomosed end-to-end to the great saphenous vein and the vena comitante of the dorsalis pedis artery. The medial plantar nerve innervating the flap was anastomosed to the deep peroneal nerve of the recipient site. A fullthickness skin graft was performed at the donor sites (Fig. 2).
Case 3

A 39-year-old man had undergone external fixation at another hospital for the management of an open comminuted fracture of the right tibia received in a traffic accident. Then, in the department of orthopedic surgery at our medical institution, the patient also underwent iliac bone grafting at the site of a bone defect caused by the comminuted fracture. However, he bone graft necrotized and progressed to osteomyelitis. Therefore, the patient was referred to us for reconstruction. At the time of referral, the patient had a $8 \mathrm{~cm} \times 12 \mathrm{~cm}$ soft tissue defect, accompanied by a bone defect approximately $8 \mathrm{~cm}$ in size. We performed debridement, including the dead bone tissue with osteomyelitis to prepare the recipient sites. We elevated an osteocutaneous free flap from the left side, involving $12 \mathrm{~cm}$ of fibula, and then inset it to the recipient site. Following the fixation of the proximal and distal part of the tibia using a screw, we performed external fixation. The artery was anastomosed end-to-side to the anterior 


\section{Fig. 3. Defect of the pretibial area}

A 39-year-old man with an open comminuted fracture of the right proximal tibia from a traffic accident. (A) Bone and soft tissue defects accompanied by osteomyelitis. (B) Elevation of a fibular osteocutaneous free flap on the contralateral side. (C) Follow-up at postoperative month 5 .
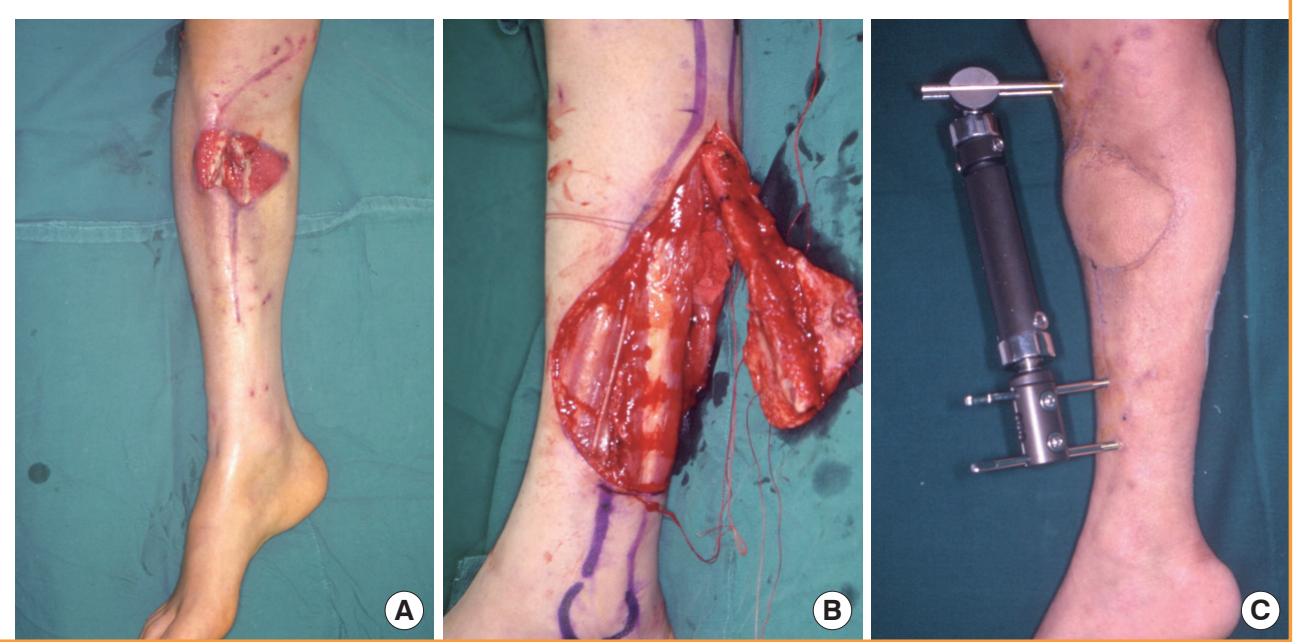

Fig. 4. Defects of the pretibial area

A 41-year-old man with an open fracture of the left tibia from a traffic accident. (A) Fixation of the fracture site with a screw and a plate following debridement. (B) Insetting of a left latissimus dorsi free flap elevated to the recipient site. (C) Photograph at postoperative month 2 .
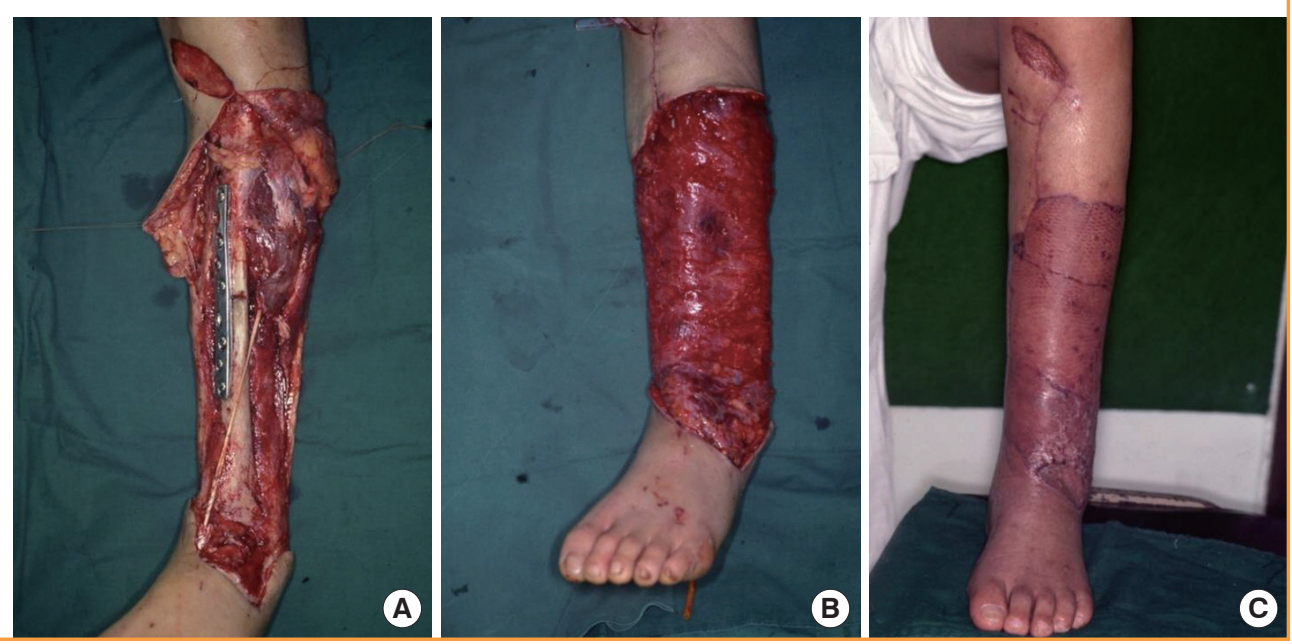

tibial artery. In addition, 2 venae comitantes were anastomosed end-to-end to the lesser saphenous vein and the vena comitante of the anterior tibial artery. A split-thickness skin graft was performed at the donor sites (Fig. 3).

\section{Case 4}

A 41-year-old man underwent open reduction and internal fixation at the department of orthopedic surgery at our medical institution to treat an open fracture of the left tibia received in a traffic accident. Afterwards, however, the patient presented with necrosis of the soft tissue over the pretibial area, accompanied by the presence of a $35 \mathrm{~cm} \times 17 \mathrm{~cm}$ soft tissue defect. The patient was therefore referred to us for further evaluation and treatment. We performed debridement in the operation room and prepared the recipient sites. Then, we elevated the left latissimus dorsi muscle free flap and inset it to the recipient site. The artery was anastomosed end-to-end to the anterior tibial artery. In ad- dition, 2 venae comitantes were anastomosed end-to-end, one each to the great saphenous vein and the vena comitante of the anterior tibial artery. A split-thickness skin graft was performed over the flap. Primary closure was performed in the donor site (Fig. 4).

\section{DISCUSSION}

The primary goal of lower extremity reconstruction is to recover function and maintain it, for which skeletal reconstruction with stable soft-tissue coverage is essential. In cases of failure in the initial reconstruction, there is the possibility that more tissue and function could be lost. It is therefore vital to make the appropriate treatment plan, and all this suggests that free flap would be the best treatment for patients with severe injuries to the lower extremities.

The free flap has been successfully used for lower extremity re- 
construction for the past 40 years. However, it has the disadvantage that necrosis of the flap is more likely to occur than it would be in another region of the body. Melissinos and Parks [3] reported that the success rate of free flap was $96.8 \%, 100 \%, 98.8 \%$, and $95.6 \%$ in the reconstruction of defects of the head and neck, the trunk, the upper extremities, and the lower extremities, respectively. Likewise, Percival et al. [4] reported values of $94 \%$, $100 \%, 91 \%$, and $85 \%$, respectively, for the same four types of defects.

In the reconstruction of lower extremity defects, however, surgeons should consider such factors as the risk of infection as well as the characteristics and size of the soft tissue defect sites. The dorsum of the foot is the area where the skin is both thin and flexible and is devoid of the subcutaneous adipose tissue. To be certain that the patients can not only wear shoes but also obtain satisfactory cosmetic outcomes, surgeons should elevate thin flaps. In cases of small dorsal foot defects, we mainly used a fascial flap. In cases of larger defects, we mainly used fasciocutaneous flaps such as an anterolateral femoral flap or scapular flap. We also used a latissimus dorsi muscle flap for reconstructing the entire dorsum of the foot or large defects. In cases of dorsal foot defects involving other adjacent regions, we performed reconstruction using a muscle flap involving the latissimus dorsi muscle and the serratus anterior muscle. Among the cases of dorsal foot defects, 2 had the concurrent presence of metatarsal bone defects, for which we used a scapular osteocutaneous free flap.

The plantar area is a weight-bearing area, and it is therefore characterized by thick skin and a larger amount of fat. Moreover, it also requires a protective sensation for weight bearing during walking. In 5 cases of reconstruction of defects of the heel and plantar forefoot, which serve as weight-bearing areas, we used a medial plantar sensory free flap providing both identical tissue material and sensory function. In the remaining 2 patients, however, the above free flap could not be used for the reconstruction surgery because the defects were of too large a size. Accordingly, in these patients, we performed reconstruction of the defects using a latissimus dorsi muscle free flap concurrently with a splitthickness skin graft. In one of 2 patients, however, a pressure ulcer of the heel occurred 1 year later. This was treated by a fullthickness skin graft harvested from the medial plantar region on the contralateral side. No complications occurred in the other patient.

The thigh is abundant with the soft tissue. Therefore, there is a remote possibility that defects of the thigh can be reconstructed with a free flap. In one of our patients, $3 / 4$ of the femur was removed due to the underlying presence of a giant cell tumor, and the defect site was implanted with hardware. Subsequent hardware exposure, then, required the soft tissue of the thigh to be reconstructed using a latissimus dorsi musculocutaneous free flap. When divided into 3 parts, the pretibial area can be reconstructed using the adjacent soft tissue in cases of proximal and middle lower leg wounds. There are many cases in which a free flap is used for the reconstruction of the distal lower leg. In our series, there were a total of 17 patients for whom pretibial defects were reconstructed. Nearly all of these cases involved the distal tibia. Of these, 10 had the concurrent presence of chronic osteomyelitis. In these cases, the bone defects occurred following debridement. Reconstruction for these was performed using a free flap involving the bone. In the remaining 7 cases, only soft tissue defects were reconstructed, all of which were large in size. Here, muscle or fasciocutaneous flaps were used for reconstruction surgery. It is commonly noted that osteomyelitis occurs in patients with severe open fractures of the tibia where the wounds have become contaminated. Inappropriate debridement could lead to the development of osteomyelitis. If there is a concurrent presence of chronic osteomyelitis, all affected bone structures should be removed and reconstruction involving bone tissue should be performed. Although still controversial, it has been recognized that, from several perspectives, such as survival, complication rates, and bone tissue healing, the muscle flap is more advantageous than the fasciocutaneous flap [5-7]. In recent years, however, it has been reported not only that there is no significant difference in surgical outcomes between the muscle flap and the fasciocutaneous flap, but also that there is no need to sacrifice muscle tissue at donor sites [8].

In cases of larger-sized defects, it would be appropriate to use a free muscle flap regardless of the defect site; the latissimus dorsi muscle, the rectus abdominis muscle, and the serratus anterior muscle being the ones primarily used. We performed reconstruction of pretibial area or dorsal foot defects using the serratus anterior muscle, the rectus abdominis muscle, or the latissimus muscle in that order for increasingly large defect sites. Muscle is rich in vascularity and thus greatly resistant to infection. Therefore, we used a muscle flap in the reconstruction of cases in which the wounds were infected, the hardware was exposed, or osteomyelitis was concurrently present. Moreover, a muscle flap has the advantage that its thickness decreases with time due to muscle atrophy.

The cases were analyzed over a long period (10 years), which meant that trends in surgery also influenced the selection of the flap. In the beginning, the scapular flap was the preferred choice when a fascial or fasciocutaneous flap was needed, but after 2000, the anterolateral thigh flap was predominantly used. When bony reconstruction was needed, the scapular osteocutaneous flap was originally used, but as time went by, it was replaced by the iliac osteocutaneous flap, and then by the fibular 
osteocutaneous flap. The authors could not find any relevance of the flap choice based on surgery trends and flap survival.

The selection of appropriate recipient vessels is an essential element for achieving successful treatment outcomes in the reconstruction of lower extremity defects using free flap [9]. The anterior tibial artery is more vulnerable to damage compared with the posterior tibial artery. It has therefore been reported that the posterior tibial artery would be more appropriate as a recipient vessel [10-12]. If there was damages to the arteries at recipient sites, however, we dissected their proximal part and anastomosed it to the arteries with normal perfusion. In two patients with arterial insufficiency, the dorsalis pedis artery served as the recipient vessel, for which an end-to-end anastomosis was performed. Godina [13] reported that end-to-side anastomosis showed superior results compared with end-to-end anastomosis. Afterwards, however, several authors have reported that there was no significant difference in flap survival between endto-side anastomosis and end-to-end anastomosis $[14,15]$. In two patients with venous thrombosis, the thrombosis occurred at the sites of anastomosis to the great saphenous vein. It has been reported that superficial veins are more vulnerable to damage compared with deep veins because of the anatomical location. In particular, a poor prognosis of the great saphenous vein has been documented [11]. In addition, it has also been reported that there is no significant difference in treatment outcomes between end-to-side anastomosis and end-to-end anastomosis. This was also seen in venous anastomosis $[14,15]$. According to Germann and Steinau [16], there was no significant correlation between the survival of the flap and the vein graft. In our clinical series of patients, however, flap survival occurred in 47 out of 48 patients who did not undergo vein grafting, yielding a success rate of $97.9 \%$. Moreover, flap survival also occurred in 3 of 4 patients who underwent vein grafting, giving a success rate of $75 \%$. When these results were analyzed with a chi-squared test $(P=0.149>0.05)$, no statistical significance was found, which led to the conclusion that no correlation existed between vein grafting and flap survival.

Our results showed that the success rate of free flap was $96.2 \%$ (50/52); this was considerably higher than other reports. That is, the success rate of free flap was reported to be $96 \%(48 / 50)$ by Basheer et al. [9], 85\% (46/54) by Percival et al. [4], 90\% (63/70) by Small and Mollan [17]. Presumably, this high success rate might be based on the mandatory use of preoperative debridement, the selection of appropriate free flap for the recipient site, and the use of appropriate, intact recipient vessels.

The authors stress that strict preparation as well as the appropriate selection of the recipient vessel were the features that led to good results. The authors made every effort to preserve as much as possible of the intact arteries that was not relevant to the defect site for circulation in the lower extremity. For this reason, in most cases, the end of the recipient artery was exposed to the defect site, and was dissected toward the proximal side. Even if the arterial flow was intact, anastomosis was performed by setting the reference point of the intact area of the vessel to a more proximal side. When the surrounding blood vessels were in poor condition, the use of the popliteal artery or the superficial femoral artery was inevitable.

As mentioned before, two anastomoses were performed for all of the veins. For the recipient vein, we tried to use one superficial vein and one deep vein whenever possible. Our aim in doing so was to drain the venous drainage of the flap to different sites. We selected the superficial veins whose ends were not exposed to the defect site whenever possible; however, as most deep veins were exposed to the defect site along with the recipient artery, we expected the deep veins to have been damaged along with the artery and applied a strict reference point for the intact vessel, as was done for arterial preparation.

\section{REFERENCES}

1. Gottlieb LJ, Krieger LM. From the reconstructive ladder to the reconstructive elevator. Plast Reconstr Surg 1994;93: 1503-4.

2. Pollak AN, McCarthy ML, Burgess AR. Short-term wound complications after application of flaps for coverage of traumatic soft-tissue defects about the tibia. The Lower Extremity Assessment Project (LEAP) Study Group. J Bone Joint Surg Am 2000;82:1681-91.

3. Melissinos EG, Parks DH. Post-trauma reconstruction with free tissue transfer: analysis of 442 consecutive cases.J Trauma 1989;29:1095-102.

4. Percival NJ, Sykes PJ, Earley MJ. Free flap surgery: the Welsh Regional Unit experience. Br J Plast Surg 1989;42:435-40.

5. Calderon W, Chang N, Mathes SJ. Comparison of the effect of bacterial inoculation in musculocutaneous and fasciocutaneous flaps. Plast Reconstr Surg 1986;77:785-94.

6. Chang N, Mathes SJ. Comparison of the effect of bacterial inoculation in musculocutaneous and random-pattern flaps. Plast Reconstr Surg 1982;70:1-10.

7. Mathes SJ, Alpert BS, Chang N. Use of the muscle flap in chronic osteomyelitis: experimental and clinical correlation. Plast Reconstr Surg 1982;69:815-29.

8. Sofiadellis F, Liu DS, Webb A, et al. Fasciocutaneous free flaps are more reliable than muscle free flaps in lower limb trauma reconstruction: experience in a single trauma center. J Reconstr Microsurg 2012;28:333-40. 
9. Basheer MH, Wilson SM, Lewis H, et al. Microvascular free tissue transfer in reconstruction of the lower limb. J Plast Reconstr Aesthet Surg 2008;61:525-8.

10. Bowen V, Manktelow RT. Complications and unsatisfactory results in the microsurgical reconstruction of lower extremities. Microsurgery 1993;14:196-202.

11. Chen HC, Chuang CC, Chen S, et al. Selection of recipient vessels for free flaps to the distal leg and foot following trauma. Microsurgery 1994;15:358-63.

12. Godina M, Arnez ZM, Lister GD. Preferential use of the posterior approach to blood vessels of the lower leg in microvascular surgery. Plast Reconstr Surg 1991;88:287-91.
13. Godina M. Preferential use of end-to-side arterial anastomoses in free flap transfers. Plast Reconstr Surg 1979;64:673-82.

14. Frodel JL, Trachy R, Cummings CW. End-to-end and endto-side microvascular anastomoses: a comparative study. Microsurgery 1986;7:117-23.

15. Samaha FJ, Oliva A, Buncke GM, et al. A clinical study of end-to-end versus end-to-side techniques for microvascular anastomosis. Plast Reconstr Surg 1997;99:1109-11.

16. Germann G, Steinau HU. The clinical reliability of vein grafts in free-flap transfer. J Reconstr Microsurg 1996;12:11-7.

17. Small JO, Mollan RA. Management of the soft tissues in open tibial fractures. Br J Plast Surg 1992;45:571-7. 